\title{
Dynamics of the MEMS Pulsed Digital Oscillator with Multiple Delays in the Feedback Loop
}

\author{
Elena Blokhina and Orla Feely \\ School of Electrical, Electronic and Mechanical Engineering \\ University College Dublin \\ Dublin, Ireland \\ blokhinaev@gmail.com
}

\author{
Manuel Domínguez \\ Department of Electronic Engineering \\ Universitat Politécnica de Catalunya \\ Barcelona, Spain
}

\begin{abstract}
In this paper we apply methods of nonlinear dynamics to examine the behavior of the pulsed digital oscillator for microelectromechanical systems (MEMS). We study the regions of existence of oscillations and demonstrate the effect on these of including additional delays into the feedback loop.
\end{abstract}

\section{INTRODUCTION}

The MEMS pulsed digital oscillator (PDO) [1] is a largesignal oscillator that utilizes a micromechanical resonator in a feedback loop of a type well known from the domain of sigma-delta modulation [2]. The general single-feedback topology of this oscillator is shown in Fig. 1. The position of the resonator is evaluated at each sampling time, and short force pulses (which can take two possible values $\{+F,-F\}$ depending on the position of the resonator) are applied to it. In the figure, $D$ is the number of delay blocks in the feedback loop and $\varepsilon$ is the sign of the feedback term. This design overcomes a number of difficulties and eliminates the impact of certain nonlinearities that beset other topologies.

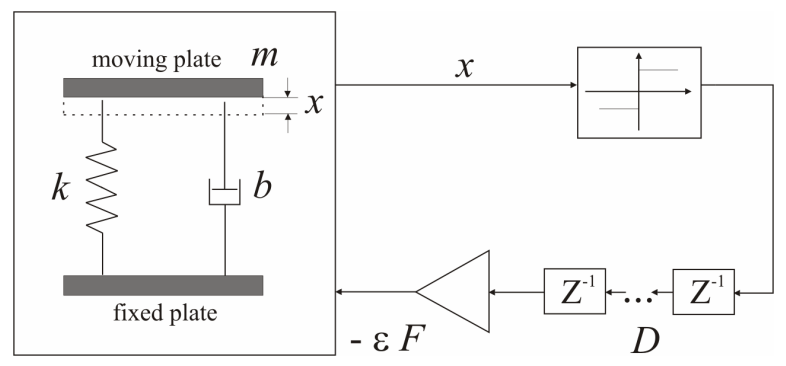

Figure 1. Topology of the pulsed digital oscillator.

Simulations and experimental verification of the PDO are carried out in [1]. It has been shown there that the plot of the oscillation frequency as a function of the natural frequency of the resonator is similar to the devil's staircase known from the domain of nonlinear dynamics. In [3], it has been shown that the PDO can maintain oscillations even for sampling frequencies below the Nyquist limit.

The dynamics of the simplest topology with one delay in the feedback loop have been studied in [4] by application of techniques of nonlinear dynamics. This work included the study of regions of admissibility of limit cycles in the plane spanned by parameters of the system (the parameter plane). The devil's staircase of [1] is obtained by taking a slice through this plane. Ref. [5] considers mechanical nonlinearity in the resonator, which causes distortion of the regions of existence of limit cycles (the tongues) in the parameter plane.

The aim of this paper is to examine the general singlefeedback topology of Fig. 1. We study the parameter plane for the cases of two and three delay blocks in the feedback loop, showing the rearrangements that are caused by the delay. In [6], it has been noted that depending on the sign of the feedback term $\varepsilon$ the PDO can operate in "reverse" mode. This mode corresponds to oscillations with small amplitude, i.e. to limit cycles near the origin. We present the planes for different signs of the feedback term and show the structure of the tongues in the regions where the PDO demonstrates the "reverse" behavior.

\section{ITERATIVE SYSTEM}

The position $x(t)$ of the resonator shown in Fig. 1 is described by the second-order differential equation

$$
m \frac{d^{2} x}{d t^{2}}+b \frac{d x}{d t}+k x=-\varepsilon F \sum_{n} \delta\left(t-t_{n}\right) \operatorname{sgn}\left(x\left(t_{n-D}\right)\right),
$$

where $m$ is the mass of the movable plate, $b$ is the damping factor, $k$ is the spring factor, $F$ is the amplitude of impulses, which affect the system at the moments $t_{n}=n T_{s}, \delta(t)$ is the Dirac delta function and $\operatorname{sgn}(x)$ is the signum function. We introduce the variable $y=-\frac{\beta}{\sqrt{1-\beta^{2}}} x-\frac{1}{\omega_{0} \sqrt{1-\beta^{2}}} v$, where

The work was supported in part by Science Foundation Ireland. 
$\beta=b /(2 \sqrt{\mathrm{km}})$ is the dimensionless damping factor, $\omega_{0}$ is the natural frequency of the resonator and $v(t)=d x / d t$.

The map describing the evolution of the values $x_{n}=x\left(n T_{s}\right)$ and $y_{n}\left(n T_{s}+\right)$ at the moments $n T_{s}$ has been obtained in [5] for the case of $D=1$, and it can be extended to arbitrary $D$ :

$$
\left(\begin{array}{l}
x_{n+1} \\
y_{n+1}
\end{array}\right)=a \mathrm{R}(2 \pi r)\left(\begin{array}{l}
x_{n} \\
y_{n}
\end{array}\right)+\left(\begin{array}{l}
0 \\
Y
\end{array}\right) \operatorname{sgn}\left(x_{n-D+1}\right)
$$

$\mathrm{R}(\theta)=\left(\begin{array}{cc}\cos \theta & -\sin \theta \\ \sin \theta & \cos \theta\end{array}\right), \quad r=T_{s} \omega_{0} \sqrt{1-\beta^{2}} /(2 \pi) \quad$ is $\quad$ the normalized sampling ratio, $a=\exp \left(-2 \pi \beta r / \sqrt{1-\beta^{2}}\right)$ is the contraction factor and $Y=\varepsilon F /\left(\omega_{0} m \sqrt{1-\beta^{2}}\right)$ is the normalized increment.

The oscillations displayed by the PDO correspond to stable limit cycles of the map (2). From the standpoint of nonlinear dynamics, it is natural to consider the rotation number defined as in [4] and discussed more generally in [7], which shows the average number of loops around the origin a trajectory makes in a single step. Note that in practice it is easier to measure the oscillation frequency, not the rotation number. In [8], it has been shown that the frequency can be easily obtained directly from PDO bitstream output. However, since we study the system as a dynamical one, we will use the rotation number introduced by (3) throughout the paper.

For a given $N$-periodic sequence of signs $\sigma_{n}$, there can be only one $N$-periodic trajectory of the system (2):

$$
\begin{aligned}
x_{n}= & \frac{Y}{\left(1-2 a^{N} \cos 2 \pi N r+a^{2 N}\right)} \times \\
\times & \left\{-\left(1-a^{N} \cos 2 \pi N r\right) \sum_{m=0}^{N-1} \sigma_{n-m-D} a^{m} \sin 2 \pi m r-\right. \\
& \left.-a^{N} \sin 2 \pi N r \sum_{m=0}^{N-1} \sigma_{n-m-D} a^{m} \cos 2 \pi m r\right\}, \\
y_{n}= & \frac{Y}{\left(1-2 a^{N} \cos 2 \pi N r+a^{2 N}\right)} \times \\
\times & \left\{-a^{N} \sin 2 \pi N r \sum_{m=0}^{N-1} \sigma_{n-m-D} a^{m} \sin 2 \pi m r\right. \\
& \left.+\left(1-a^{N} \cos 2 \pi N r\right) \sum_{m=0}^{N-1} \sigma_{n-m-D} a^{m} \cos 2 \pi m r\right\} .
\end{aligned}
$$

If the $N$-periodic sequence generated by (3) satisfies the condition

$$
\operatorname{sgn} x_{n}=\sigma_{n},
$$

then it is a limit cycle [4]. The sequence of signs in (3) is generated as follows: $\sigma_{n}=\operatorname{sgn}\left(\cos \left(2 \pi \rho n+\phi_{0}\right)\right)$, where $\rho$ is the ratio of two integers $\rho=M / N$ with the greatest common divisor g.c.d. $(M, N)=1$ and $\phi_{0} \in[0,2 \pi)$. Equations (3) and (4) allow us to find the domain of existence of a particular limit cycle with rotation number $\rho$ on the $(\beta, r)$ parameter plane.

\section{DYNAMICS OF THE PDO WITH SEVERAL DELAYS IN THE FEEDBACK LOOP}

The dynamics of the system (2) in the case of one delay have been studied in $[1,4,5]$. The aim of this section is to study changes in the behavior of the system when the number of delays is increased.

As noted in [1], energy can be put into or extracted from the resonator. When the velocity of the resonator is positive, applying a positive impulse leads to an increase in the kinetic energy of the resonator. On the other hand, when the velocity is negative, applying a positive impulse will decrease the energy.

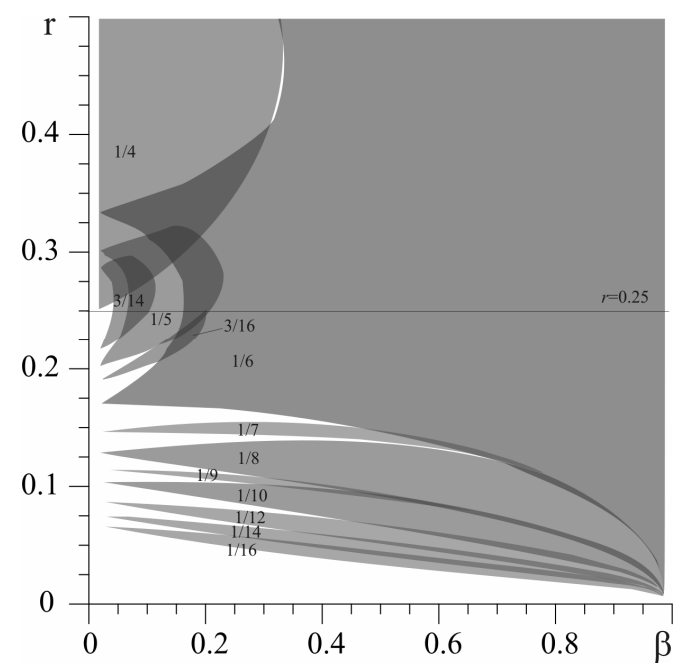

(a)

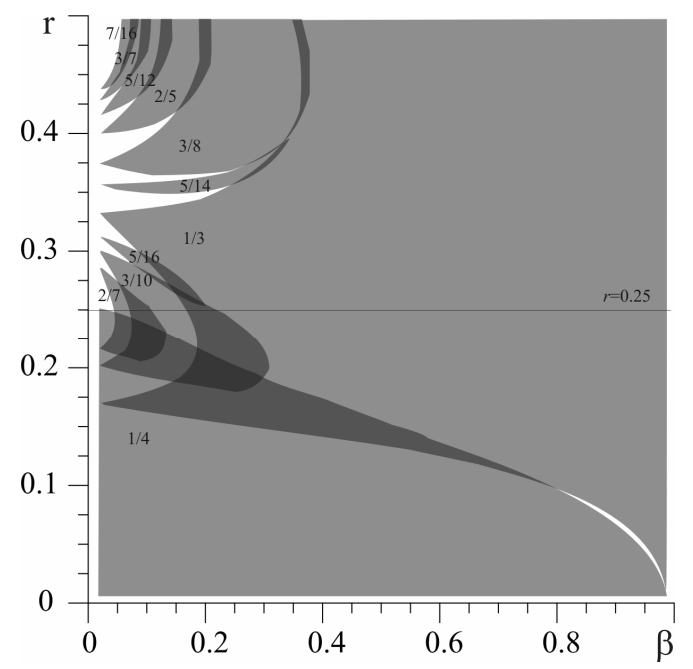

(b)

Figure 2. Tongues on the parameter plane for $\varepsilon=(a)+1$; (b) -1 . The rotation number of the corresponding limit cycle is indicated for each tongue. 
Sustained oscillations in the PDO are reached if the feedback sign $\varepsilon$ is selected correctly. In the case of one delay in the feedback loop, at the sampling ratio $r>0.5$ one has to switch the sign $\varepsilon=+1$ to $\varepsilon=-1$ in order to preserve the qualitative behavior of the system.

Fig. 2 illustrates tongues on the plane of parameters $(\beta, r)$ in the case of two delays in the feedback loop. The tongues shown in Fig. 2(a) were calculated with $\varepsilon=+1$ over the entire region of the sampling ratio $0<r<0.5$, whereas the tongues in Fig. 2(b) have $\varepsilon=-1$. Each tongue in the figures corresponds to the domain of existence of a particular limit cycle with the given rotation number.

Examining first Fig. 2(a), where $\varepsilon=+1$, we see that for $r<0.25$ and $\beta$ close to 0 (as will be the case in practice with a high-quality resonator) the tongues are very narrow and close to the value $r=\rho$. This means that the (normalized) frequency of the output of the system is very close to the (normalized) natural frequency of the resonator. Many MEMS sensors exploit the variation of that natural frequency with environmental conditions, so the fact that the output frequency follows that natural frequency permits the use of this PDO in such sensors. The non-zero width of the tongues limits resolution in a highly nonlinear fashion (the devil's staircase), but for very small $\beta$ this loss of resolution is within acceptable levels.

For $r>0.25$, however, we see that even for very low values of $\beta$ the behaviour is no longer suitable for such applications. Only a small number of low-order limit cycles appear, and they persist over wide intervals of $r$. There is also considerable overlap between the tongues, which means that the rotation number depends on initial conditions.

In addition, the amplitudes of oscillations for $r>0.25$ are considerably smaller than for $r<0.25$. This is illustrated by the simulation results presented in Fig. 3, which shows the 48-cycle at $\beta=0.001$ and $r=0.23$ and the 4-cycle (with a much smaller amplitude) at the same value of dissipation and $r=0.26$. Both values of the sampling ratio are close to the "boundary" value $r=0.25$.

Note that the amplitude of oscillations decreases gradually and tends to zero around the value $r=0.25$. Fig. 4 shows the amplitude of a limit cycle as a function of the normalized sampling ratio $r$. The inset of the figure shows the magnified view of the plot near the value $r=0.25$.

In general, we observe the normal mode (large amplitude oscillations) and "reverse" mode (very small amplitude oscillations) depending on values of the sampling ratio. The "reverse" mode behavior has been described in [6] for the case of one delay. It has been shown there that one can control oscillations by switching the sign of the feedback, i.e. turning the PDO to the "reverse" mode and back. For the topology with $\varepsilon=-1$, the region of the "reverse" mode is $0<r<0.25$, as is evident from Fig. 2(b). Comparing Fig.2(a) and Fig. 2(b), we see that one has to change the sign of $\varepsilon$ at $r=0.25$ to keep the desirable qualitative behavior. As a result, the entire plane of parameters consists now of two parts, as shown in Fig. 5.

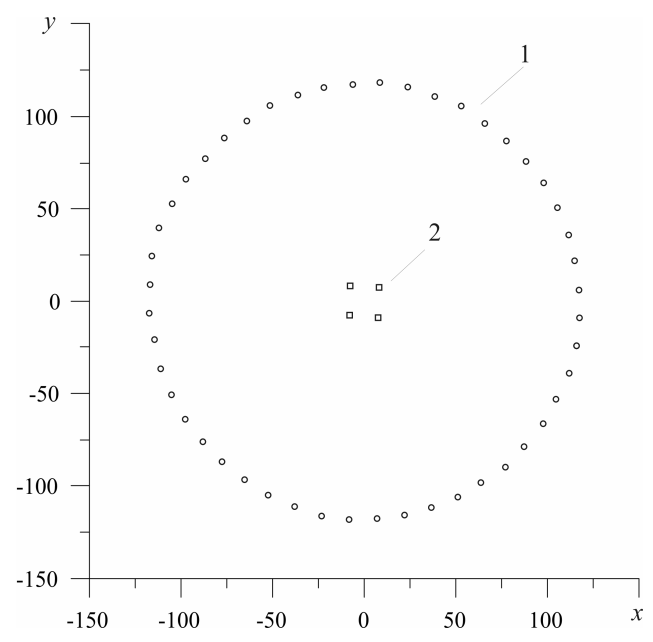

Figure 3. The cycle marked 1 is the 48 -cycle observed in the system at $\beta=0.001$ and $r=0.23$, and that marked 2 is the 4-cycle observed at $\beta=0.001$ and $r=0.26$. The sign of the feedback $\varepsilon=+1$, and $D=2$.

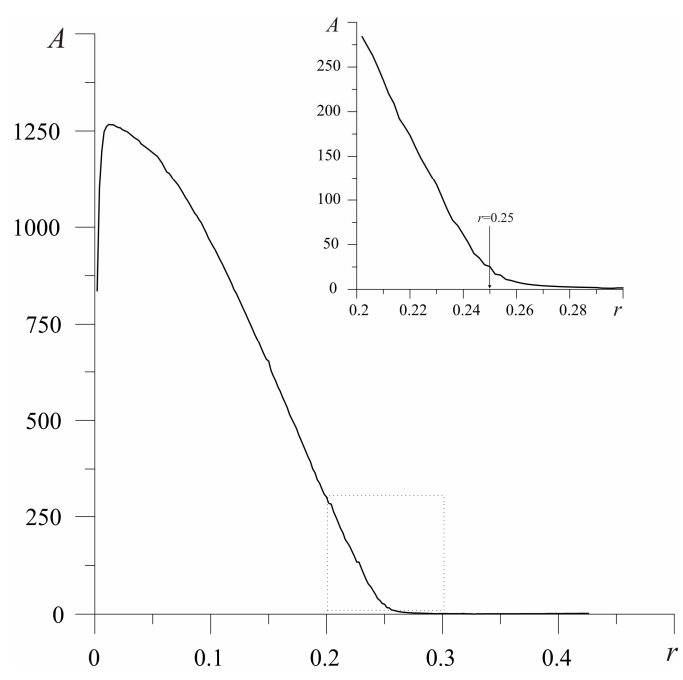

Figure 4. Amplitude of a limit cycle as a function of the normalized sampling ratio $r$. The inset shows a magnified version. $D=2$.

A similar situation takes place if three delays are included in the feedback loop. Fig. 6(a) shows tongues on the parameter plane for $\varepsilon=+1$ and $D=3$. The region of the sampling ratio $1 / 6<r<1 / 3$ now corresponds to the "reverse" mode of the PDO. One has to switch the sign from $\varepsilon=+1$ to $\varepsilon=-1$ at $r=1 / 6$ and back to $\varepsilon=+1$ at $r=1 / 3$ to preserve the qualitative behavior of the system. Fig. 6(b) shows tongues on the parameter plane when this switch is implemented. 


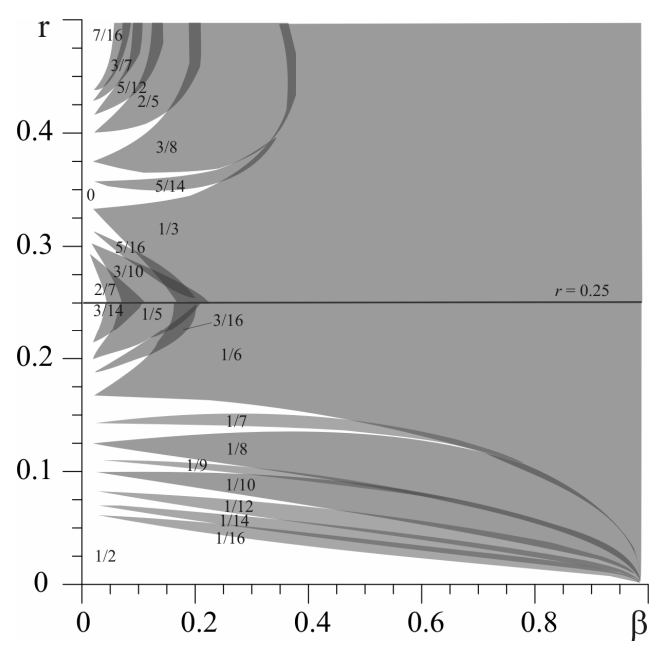

(a)

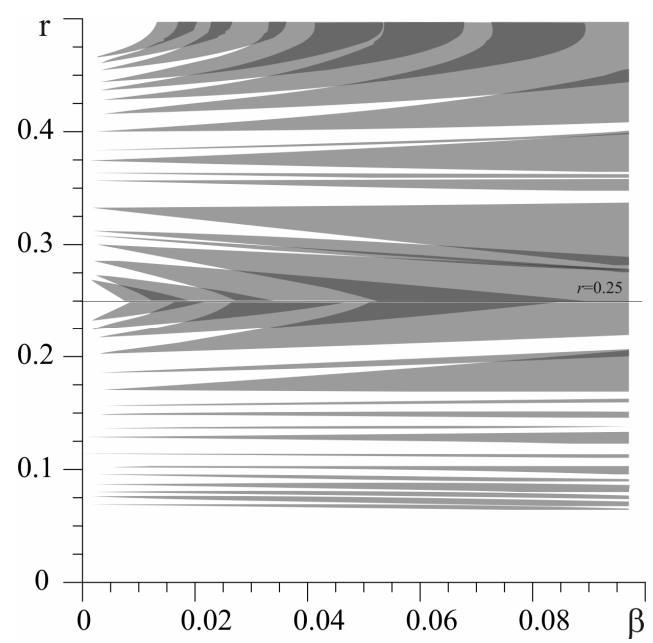

(b)

Figure 5. (a) Tongues on the parameter plane for $D=2$. The sign of the gain in the feedback loop is switched from $\varepsilon=+1$ to $\varepsilon=-1$ at $r=0.25$; (b) Magnified view of plot (a), the tongues correspond to the rotation numbers $\rho=N / M$ where $3 \leq M \leq 16$, g.c.d. $(M, N)=1$.

\section{CONCLUSION}

In this paper, we have studied the regions of existence of limit cycle oscillations in the parameter plane for the MEMS PDO with two and three delays in the feedback loop, highlighting differences with the standard one-delay case. We presented plots for different signs of the feedback term, and showed the structure of tongues and the nature of the oscillations in regions where the PDO demonstrates "reverse" behavior.

\section{REFERENCES}

[1] M. Dominguez, J. Pons-Nin, J. Ricart, A. Bermejo, E.F. Costa, and M. Morata, "Analysis of the $\Sigma \Delta$ pulsed digital oscillator for MEMS", IEEE Trans. Circuits Syst. I, Reg. Papers, vol. 52, pp.2286-2297, 2005.

[2] S.R. Norsworthy, R. Schreier, and G.C. Temes, Eds., "Delta-sigma Data converters: Theory, Simulation and Disign", New-York: IEEE Press, 1996.

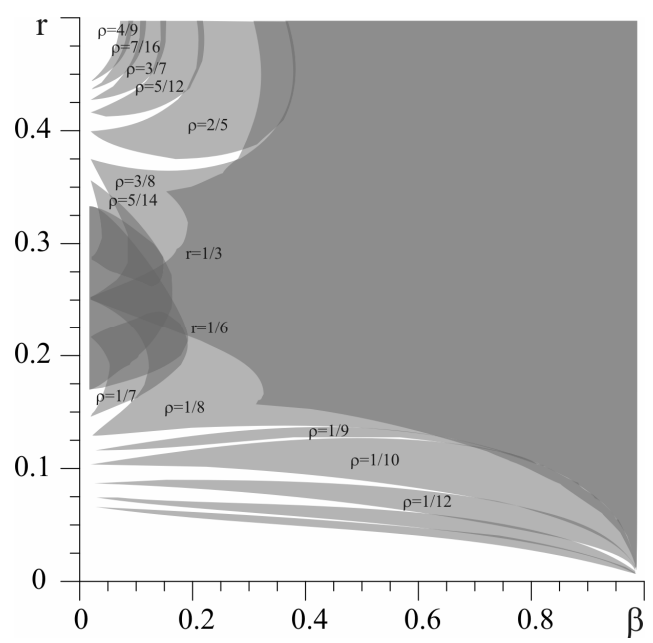

(a)

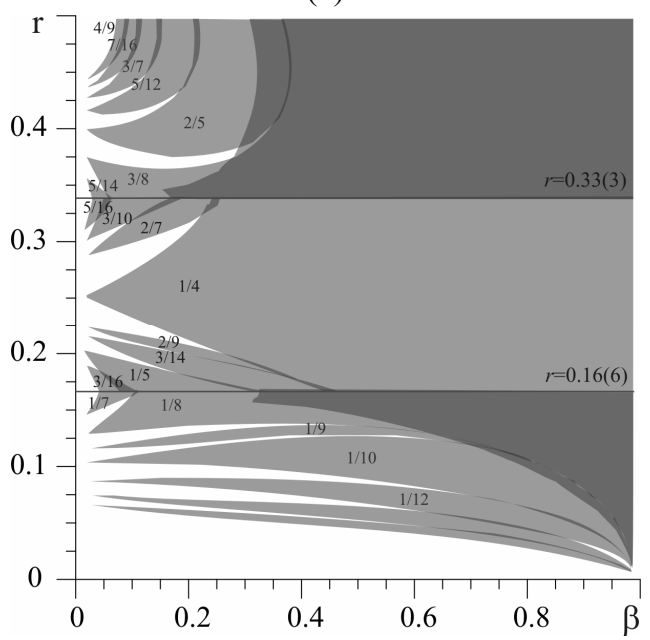

(b)

Figure 6. Tongues on the parameter plane, $D=3$. In (a) $\varepsilon=+1$ over the region $0<r<0.5$, while in (b) $\varepsilon$ is switched from +1 to -1 at $r=1 / 6$ and back to +1 at $r=1 / 3$.

[3] M. Dominguez, J. Pons-Nin, J. Ricart, and E. Figueras, "The MEMS pulsed digital oscillator (PDO) below the Nyquist limit", Sensors and Actuators, vol. A136, pp. 690-696, 2007.

[4] A. Teplinsky, and O. Feely, "Limit Cycles in a MEMS Oscillator", IEEE Trans. Circuits Syst. II. Express Briefs, vol. 55, pp. 882-886, 2008.

[5] E. Blokhina, and O. Feely, "A kicked oscillator as a model of a pulsed MEMS system", Int. Journal of Bifurcation and Chaos, vol. 19, no 1, 2009.

[6] M. Dominguez, J. Pons, and J.Ricart, "Application of Pulsed-Digital Oscillators in 'reverse mode' to eliminate undesired vibrations in highQ MEMS resonators", IEEE Int. Simp. On Circuits and System, ISCAS 2007, New Orleans, USA, May 27-30, pp. 925-928, 2007.

[7] R. Hilborn, "Chaos and Nonlinear Dynamics: An Introduction for Scientists and Engineers", Oxford, U.K.: Oxford University Press, 1996.

[8] M. Dominguez, J. Pons-Nin, and J. Ricart, "General Dynamics of Pulsed Digital Oscillators", IEEE Trans. Circuits Syst. I. Regular Papers. Vol. 55, pp. 2038-2050, 2008. 